\title{
Bases conceptuales y metodológicas para el estudio de los espacios fluviales urbanos. Un estudio de caso en Andalucía
}

\section{Conceptual and methodological bases for the study of urban river spaces. A case study in Andalusia}

\author{
David González Rojas ${ }^{1}$
}

\begin{abstract}
RESUMEN
El presente trabajo tiene como objetivo poner de manifiesto la necesidad de trabajar en distintas escalas, espaciales y temporales, y el reconocimiento de las relaciones entre los ríos y el patrimonio a la hora del estudio de los espacios fluviales urbanos para poder entender su identidad y carácter.

Para ello se ha realizado un estudio de los espacios fluviales urbanos que incluye dos nuevos conceptos que hasta ahora no habían sido estudiados de forma conjunta: el análisis en distintas escalas y el estudio de los aspectos materiales e inmateriales.

La mayoría de las actuaciones en cauces urbanos han generado transformaciones rápidas e intensas que provocan la pérdida de esta identidad. Los problemas, insuficiencias y oportunidades específicos son la clave para la definición de procedimientos metodológicos comunes que tengan en cuenta los componentes ambientales, culturales, patrimoniales y sociales.

Esta metodología permite el análisis multidisciplinar de los espacios fluviales urbanos, con el objetivo de analizar estos espacios como garantía de su conservación y puesta en valor dentro de su ámbito urbano.

Por último, cabe señalar que las múltiples funciones de los espacios fluviales hacen que profundizar en su conocimiento y en los procesos que han llevado a la pérdida de los mismos sea una necesidad a la hora de conservar y mejorar nuestros ríos. En
\end{abstract}

1 Consultor ambiental. Sevilla. davidgonzalezrojas@gmail.com ORCID iD: http://orcid.org/ 0000-0003-2326-5178

Copyright: @ 2017 CSIC. Este es un artículo de acceso abierto distribuido bajo los términos de la licencia de uso y distribución Creative Commons Attribution (CC-by) España 3.0. 
atención al tratamiento histórico dado a la mayoría de espacios fluviales en Andalucía, es necesario plantearse una serie de cuestiones sobre las que avanzar en los siguientes años: ¿es posible recuperar los espacios fluviales eliminados? ¿funcionan realmente las medidas de conservación actualmente implementadas? etcétera. Éstas y otras preguntas engloban en su concepción y en sus posibles respuestas, una amplia variedad de discusiones relativas a los espacios fluviales y su conservación, así como hacia el modelo de crecimiento que se persigue, debiéndose recuperar y conservar los valores esenciales que los espacios fluviales andaluces han tenido históricamente en el paisaje, el patrimonio, las costumbres y formas de vida.

Palabras Clave: espacios fluviales urbanos; Andalucía; planeamiento urbanístico; patrimonio; análisis multiescalar.

\section{Abstract}

This paper aims to highlight the need of working into different scales, spatial and temporal, and the identification of relationships between rivers and heritage at the time of study urban river spaces in order to understand their identity and character.

For this purpose, a research of urban-river areas has beendeveloped including two new concepts that had not been studied together until now: analysis in different scales and study of both material and immaterial aspects.

Most of the already taken measures in urban river channels have generated intense and rapid transformations that indeed result in the loss of this identity. The problems, weaknesses and specific opportunities are the key to defining common methodological procedures which take into account environmental, cultural, economic and social components.

This methodology allows the multidisciplinary analysis of the urban river spaces, in order to analyze them as a guarantee of their own conservation, and also put them in value within its urban scope.

Lastly, it must be remarked that the multiple functions fluvial spaces have, make deepening in its knowledge and the processes that have given raise to their loss a need in order to maintain and improve our rivers. Regarding the historical treatment given to the majority of the fluvial spaces in Andalusia, it is necessary to rethink a series of questions for the following years: Is it possible to recover the fluvial spaces that have disappeared? Is the conservation measures currently implemented really working? Etc. These, and other questions, encompass a great variety of discussions regarding fluvial spaces and its preservation, coupled with the model of growth that is aimed at. Thus, it is paramount to recover the essential values that fluvial spaces have had historically in our landscape, patrimony, costumes and ways of living.

KEY WORDS: river urban spaces; Andalusia; urban planning; heritage; multi-scalar analysis.

Cómo citar este artículo/Citation: González Rojas, David (2017): "Bases conceptuales y metodológicas para el estudio de los espacios fluviales urbanos. Un estudio de caso en Andalucía", Estudios Geográficos, LXXVIII/283, pp. 657-679 


\section{INTRODUCCIÓN.}

Los espacios fluviales urbanos son entornos tensionados y de difícil coexistencia. Destaca por un lado, el carácter cambiante e irregular del río, en buena parte generado por los cambios de trazado, naturales o antrópicos, como consecuencia de las avenidas importantes; y, por otro, el carácter permanente y más o menos uniforme de los elementos urbanos. Los usos del suelo y las edificaciones crean estructuras permanentes con límites fijos y estables. Por ello, la ordenación de estos espacios fluviales necesita un cierto margen de flexibilidad para prevenir posibles cambios futuros del río.

Uno de los problemas encontrados en el análisis de los espacios fluviales urbanos es la delimitación de los distintos ámbitos de estudio. En teoría, el análisis de los factores que configuran el espacio fluvial urbano da lugar a un ámbito homogéneo, pero en ocasiones estos límites se muestran de forma difusa. Otras veces se puede pensar que cuanto mayor sea la entidad del cauce, mayores son las áreas homogéneas que guardan relación con el mismo, pero esto no siempre es así. Por lo tanto, los límites a definir son abstractos y físicos, desdibujados y ambiguos, no basados en límites preestablecidos. En algunos casos es suficiente con estudiar el cauce y su entorno urbano más inmediato, en otras, es necesario hacer un estudio a mayor escala, incluso a nivel de cuenca.

Con el presente trabajo se pone de manifiesto la necesidad de trabajar en distintas escalas, espaciales y temporales, y el reconocimiento de las relaciones entre los ríos y el patrimonio a la hora del estudio de los espacios fluviales urbanos para poder entender su identidad y carácter. Por lo tanto, el presente trabajo incluye dos nuevos conceptos a la hora del estudio de los espacios fluviales urbanos que hasta ahora no habían sido estudiados de forma conjunta: el análisis en distintas escalas y el estudio de los aspectos materiales e inmateriales.

\section{Aproximación al estudio de los espacios fluviales urbanos.}

\subsection{Enfoque ecosistémico.}

El enfoque ecosistémico, nacido del pensamiento sistémico, se basa en la aplicación de métodos científicos adecuados centrados en los niveles de organización biológica que abarcan los procesos, las funciones y las interacciones esenciales entre los organismos y su ambiente, y que reconoce a los humanos, con su diversidad cultural, como un componente integrante de los ecosistemas. 
Los geomorfólogos han sido pioneros en los estudios sobre los medios de los grandes ríos (Leopold y Wolman, 1957), introduciendo la noción de sistema fluvial. Paralelamente, los ecólogos reconocen que la noción de ecosistema como conjunto unitario es difícilmente aplicable a los sistemas fluviales por su particular configuración longitudinal, y proponen, desde la Ecología, el concepto de continuum fluvial (Vannote et al., 1980), partiendo del supuesto de que las variables geofísicas en un sistema fluvial presentan una gradación constante desde el nacimiento hasta la desembocadura. Las comunidades de organismos vivos se suceden a lo largo del curso del río de manera que se reduce al mínimo la pérdida de energía.

En los años ochenta aparece un nuevo concepto, el de hidrosistema fluvial (Amorós y Petts, 1993), definido como una porción del espacio (cuenca vertiente) donde está superpuesta una parte de la atmósfera, de la superficie del suelo y del subsuelo en tres dimensiones y a través de las cuales los flujos hídricos están sometidos a unas formas particulares de circulación. Según esta noción, los ríos pueden considerarse como sistemas complejos en cuatro dimensiones (longitudinal, transversal, vertical y temporal), constituidos por ecosistemas interactivos.

\subsection{Aproximación desde la ordenación del territorio y el urbanismo.}

El territorio se configura mediante procesos complejos en los que intervienen múltiples agentes de origen natural y antrópico. El término ordenación del territorio expresa un concepto muy amplio y difícil de reducir a una definición precisa, aunque siempre girando en torno a tres elementos: las actividades humanas, el espacio donde se ubican y el sistema que entre ambos configuran. Básicamente ordenar el territorio significa identificar, distribuir, organizar y regular las actividades humanas en ese territorio de acuerdo con ciertos criterios y prioridades.

A un nivel de mayor detalle, el urbanismo comenzó siendo una teoría compleja que captó el interés, desde el primer momento, de los estudiosos de la ciudad y acabó siendo una disciplina que aglutina una suma de conocimientos sustanciales relacionados con la construcción y conservación de las ciudades, y con el estudio de las relaciones sociales, económicas y ambientales que tienen lugar dentro del fenómeno urbano.

Si bien es clara la dimensión de los cursos fluviales como elemento estructurante del territorio, la situación en las ciudades es especialmente elocuente, ya que es en la ciudad donde la tensión entre el espacio fluvial y el espacio urbano adquiere su mayor expresión (García, 2002). 
En cuanto al estudio de los espacios fluviales urbanos desde el punto de vista de la ordenación del territorio y el urbanismo, algunos autores analizan la influencia de los espacios fluviales en la organización de las ciudades y el paisaje (Bravard et al., 1995; Calvo, 1997; Moral, 1997; Pellicer, 2002; Terrin, 2014; etcétera); en contraposición de otros estudios cuyo objetivo es el análisis de los efectos del espacio urbano sobre el espacio fluvial (Sala y Inbar, 1992; González y Lastra, 2007; etcétera). Por último, otros autores se centran en las oportunidades de recuperación de estos espacios y su beneficio al medio urbano (Wade, 1997; Riley, 1998; Ollero y Ureña, 2000; Ollero, 2007; Ureña, 2000; García, 2002; etcétera).

\subsection{Aproximación histórica y patrimonial.}

No se puede entender un espacio fluvial urbano sin conocer su historia, es decir, las principales dinámicas ecológicas, socio-económicas y culturales del pasado y del presente que han llevado a la creación de los espacios actuales (Zoido, Rodríguez y Ramírez, 2010). Numerosos trabajos abordan el análisis de los espacios fluviales urbanos integrando la aproximación natural con la histórica y patrimonial. Algunos autores realizan un análisis de la evolución de las riberas urbanas como consecuencia de las distintas acciones históricas que sobre ellas se han llevado a cabo (Calvo, 1997; Carmona, 1997; Díaz y Almoguera, 2013; etcétera). En el complejo proceso evolutivo hidrogeomorfológico de los espacios fluviales urbanos intervienen variables tanto externas (climatológicas, estado de la cuenca, etcétera) como internas (actuaciones del hombre).

De estos estudios se concluye que durante una larga etapa de la historia el crecimiento de las ciudades dio la espalda a los cursos fluviales. Los ríos significaban una amenaza para la población por el peligro de inundación, por lo que acababan siendo desplazados o canalizados, destruyéndose sus ecosistemas y la vida natural de los mismos. Las soluciones defensivas y la ordenación urbana han sido distintas según las épocas y según las características propias de cada ciudad.

\section{Concepto y DELIMITACiÓN DEL ESPACIO FLUVIAL URBANO}

El término fluvial se utiliza en la geografía y en ciencias de la Tierra para referirse a los procesos asociados a los ríos y arroyos, y a los depósitos y relieves creados por ellos. Se puede delimitar el espacio fluvial mediante diversos 
criterios, partiendo de fundamentos ecológicos, hidráulicos y/o administrativos. Anteriormente se han comentado los conceptos de sistema fluvial, hidrosistema fluvial o continuum fluvial, empleados por geomorfólogos y ecólogos; a continuación se describe el espacio fluvial según criterios administrativos.

Según la Ley de aguas del estado español (Real Decreto Legislativo 1/2001, de 20 de julio, por el que se aprueba el texto refundido de la Ley de aguas) se entiende por riberas las fajas laterales de los cauces públicos situadas por encima del nivel de aguas bajas, y por márgenes los terrenos que lindan con los cauces, entendido éste como el terreno cubierto por las aguas en las máximas crecidas ordinarias. La ley subdivide a la vez el dominio público hidráulico en una zona inundable ordinaria, o sea, aquella que es ocupada en un momento u otro, de media, como mínimo cada 10 años; una zona de servidumbre, de 5 metros de anchura, para el uso público, que a la vez queda incluida a la zona de policía, de anchura superior, 100 metros. Esta delimitación, que podría facilitar la aplicación de una política efectiva de protección de los ríos, está aún pendiente de fijar en la mayoría de tramos fluviales.

En conclusión, la definición de cauce es relativamente sencilla, pues se trata de la franja de terreno cubierta por las aguas en las máximas crecidas ordinarias. Ahora bien, en su determinación influyen tan variadas circunstancias que pueden llegar a convertirse en una cuestión conflictiva; todo ello sin olvidar que las zonas contiguas sometidas a limitación, llamadas de servidumbre y policía, comienzan a cuantificarse en función de la exacta delimitación del cauce. Tal es el caso que en la propia legislación se indica que para determinar la franja de terreno cubierta por las aguas en las máximas crecidas ordinarias se atenderá no solo a criterios hidrológicos, sino también a otras características, como las geomorfológicas, las ecológicas y referencias históricas disponibles (Real Decreto 9/2008, de 11 de enero, por el que se modifica el Reglamento del dominio público hidráulico, aprobado por el Real Decreto 849/1986, de 11 de abril). De esta manera, se da cumplimiento a las exigencias de la Directiva 2007/60/CE del Parlamento Europeo y del Consejo, de 23 de octubre de 2007, relativa a la evaluación y gestión de los riesgos de inundación, que determina que los estados miembros deben incorporar políticas sobre gestión del riesgo de inundaciones que garanticen al máximo la seguridad de los ciudadanos, adoptando criterios adecuados de usos del suelo, y que permitan la laminación de caudales y de carga sólida transportada ampliando, en la medida de lo posible, el espacio fluvial disponible. Además, mediante dicha modificación del Reglamento del dominio público hidráulico se crea el Sistema nacional de cartografía de zonas inundables como elemento básico en la planificación territorial para la identificación y gestión adecuada de las zonas inundables. 
Por otro lado, hay que hacer mención al Proyecto Linde puesto en marcha en 1993 por el entonces Ministerio de Obras Públicas, Transportes y Medio Ambiente con el objetivo de delimitar y deslindar físicamente las zonas del dominio público hidráulico presionadas por intereses de cualquier tipo, que corren riesgo cierto de ser usurpadas, explotadas abusivamente o degradadas por falta de una respuesta contundente y reglamentada de la Administración.

Si a los criterios administrativos se incluyen los ecológicos e hidráulicos, el concepto de espacio fluvial supera ampliamente el dominio público hidráulico de la Ley de aguas, ya que debe incluirse el área donde acontecen todos los procesos relacionados con el funcionamiento del río: el valle fluvial por donde transcurre el río, las terrazas fluviales que reflejan la actividad geomorfológica del río, los bosques de ribera adyacentes y que dependen del agua del río, el acuífero subterráneo con el cual está conectado, etcétera.

Surge entonces un concepto de mayor extensión, el territorio fluvial o territorio de movilidad fluvial, establecido de forma consensuada en la Estrategia nacional de restauración de ríos. El territorio fluvial puede definirse como el terreno, espacio o paisaje dominado por un sistema fluvial. Es un espacio del río, que incluye el cauce, el corredor ribereño y, total o parcialmente, la llanura de inundación que permite el desarrollo de la dinámica natural (Ollero et al., 2011).

En el ámbito urbano, debido a la urbanización, las defensas de las márgenes y, en consecuencia, a la alteración de la dinámica natural, no es posible aplicar el concepto de territorio fluvial. En la mayoría de los casos ni siquiera existe disponible una franja de 5 metros de anchura para el uso público (zona de servidumbre). Es necesario, por tanto, definir un nuevo concepto para referirse al espacio fluvial en el ámbito urbano. El espacio fluvial urbano o espacio fluviourbano es el definido por el cauce del río u obra de protección (encauzamiento, canalización, etcétera), sus márgenes y el entorno inmediato que permite caracterizar las relaciones río-ciudad. Esta definición recoge espacios centrales dentro del entorno urbano, en contacto directo con el suelo urbano o a cierta distancia del núcleo de población, siempre que existan elementos históricos que hayan influido en el crecimiento y la evolución de la ciudad.

\section{Propuesta metodológica para el estudio de los espacios fluviales urbanos.}

La propuesta metodológica que se expone a continuación es de carácter multidisciplinar y pretende recoger los fundamentos conceptuales y metodológicos más significativos de las distintas disciplinas o aproximaciones desde las que es posible encarar el estudio de los espacios fluviales urbanos. 
La complejidad de las relaciones río-ciudad requiere procedimientos a su vez complejos para concebir y verificar la hipótesis de partida, utilizando mecanismos dinámicos de ida y vuelta, anticipando desde el comienzo las hipótesis y verificándolas o corrigiéndolas permanentemente.

El desarrollo del estudio parte del interés por las circunstancias que generan la pérdida de identidad y carácter de los espacios fluviales urbanos. Los problemas, insuficiencias y oportunidades específicos son la clave para la definición de procedimientos metodológicos comunes que tengan en cuenta los componentes ambientales, culturales, patrimoniales, sociales, etcétera.

El objetivo de la propuesta metodológica es elaborar un procedimiento general para la incorporación de la identidad y carácter de los espacios fluviales urbanos en la recuperación de los mismos. En la práctica, la propuesta pretende establecer los mecanismos e instrumentos básicos que permitan:

- La adecuada contextualización de las intervenciones y su articulación con otros instrumentos de planificación.

- El reconocimiento y caracterización de los espacios fluviales urbanos en toda su complejidad y amplitud (recursos ambientales, sociales, culturales, patrimoniales, económicos, simbólicos, escénicos, etcétera).

- La identificación de los elementos que deben ser preservados para evitar la alteración de la identidad y carácter de los espacios fluviales urbanos.

- El reconocimiento de los factores y procesos con mayor incidencia en la conformación del espacio fluvial urbano.

- La eliminación o minimización de las actividades conflictivas o banalizadoras, así como la integración de los nuevos elementos y actuaciones a implantar en los espacios fluviales urbanos.

- El establecimiento de los mecanismos necesarios para el seguimiento de las intervenciones de recuperación.

A través del proceso de evaluación de la identidad y carácter se pretende establecer la fragilidad de un determinado espacio fluvial urbano ante posibles cambios o intervenciones que pudieran incidir sobre sus características claves, así como sobre aquellos valores o potencialidades identificados durante la fase de caracterización. En este sentido, la evaluación constituye un diagnóstico general sobre el estado de la identidad y carácter de un determinado ámbito fluvial urbano. Sobre dicho diagnóstico pueden sustentarse posteriormente las propuestas, medidas y criterios que se adopten en relación con la protección, gestión o recuperación de los recursos y elementos que configuran el espacio fluvial urbano. Igualmente, la evaluación de la identidad y carácter permite valorar el impacto de actuaciones concretas en el estado actual de estos entornos. 
Una vez introducido el marco ideológico del trabajo, se llega a un punto de la investigación en el cual se hace necesario introducir un campo de reflexión concreto. Andalucía aporta una situación común dentro de la evolución de las relaciones río-ciudad en las urbes occidentales, en la que ha habido un proceso de progresivo sometimiento de los cauces. Sin embargo, en esta región se produce una serie de peculiaridades que justifican la investigación: el uso desde la antigüedad de los valles fluviales como vías de comunicación y poblamiento, la diversidad climática y geológica dentro de la región que influye en una amplia variedad tipológica de ríos y, una baja intervención en los cauces pese a la expansión urbana desmesurada de las últimas décadas.

\subsection{Análisis multiescalar.}

Habitualmente en este tipo de estudios se trabaja en una sola escala, obteniendo resultados parciales. Para caracterizar el espacio fluvial urbano es necesario realizar un estudio a distintas escalas para poder entender su identidad y carácter. A cada escala y a cada planteamiento metodológico corresponde por lo tanto una descripción diferente del espacio fluvial urbano. Para ello, se ha de tener en cuenta dos concepciones de la escala.

La primera, la escala espacial, en línea con la escala cartográfica, realizando un análisis a partir de tres escalas diferentes. El objetivo que se persigue con el análisis es obtener una imagen global del espacio fluvial urbano y de sus potencialidades y problemas. De esta forma se pueden establecer directrices de protección e intervención y proponer mejoras para la futura ordenación, tanto a nivel local como en ámbitos homogéneos.

En el ámbito más extenso, la caracterización de los espacios fluvio-urbanos se ha de realizar a nivel regional. Se trata del nivel de análisis que permite una visión global de los espacios fluviales urbano dentro de una región determinada.

La metodología utilizada para la identificación del espacio fluvial urbano se basa en la individualización por separado de cada uno de los elementos que lo conforman: el espacio fluvial y el espacio urbano. En primer lugar se procede a la definición de la red hidrográfica de referencia. La red hidrográfica definida debe servir para una doble finalidad: por un lado, debe ser tal que sobre ella se puedan realizar todos los pasos necesarios para la identificación de los espacios fluviales urbanos y, por otro, debe quedar lo suficientemente definida como para delimitarlos con corrección cartográfica. Una vez definida la red hidrográfica de referencia se procede a identificar el espacio urbano. La incidencia de la utilización de un tipo de cartografía de usos del suelo determinada está, por un 
lado, en la precisión de los resultados obtenidos y, por otro, en el método utilizado para identificar los espacios fluviales urbanos a partir de esta información.

El proceso posterior de identificación de los espacios fluviales urbanos da lugar a una red de drenaje más sencilla que facilita la comprensión y visualización de los resultados de la identificación.

\section{FIGURA 1}

DELIMITACIÓN DEL ESPACIO FLUVIAL URBANO EN ANDALUCÍA

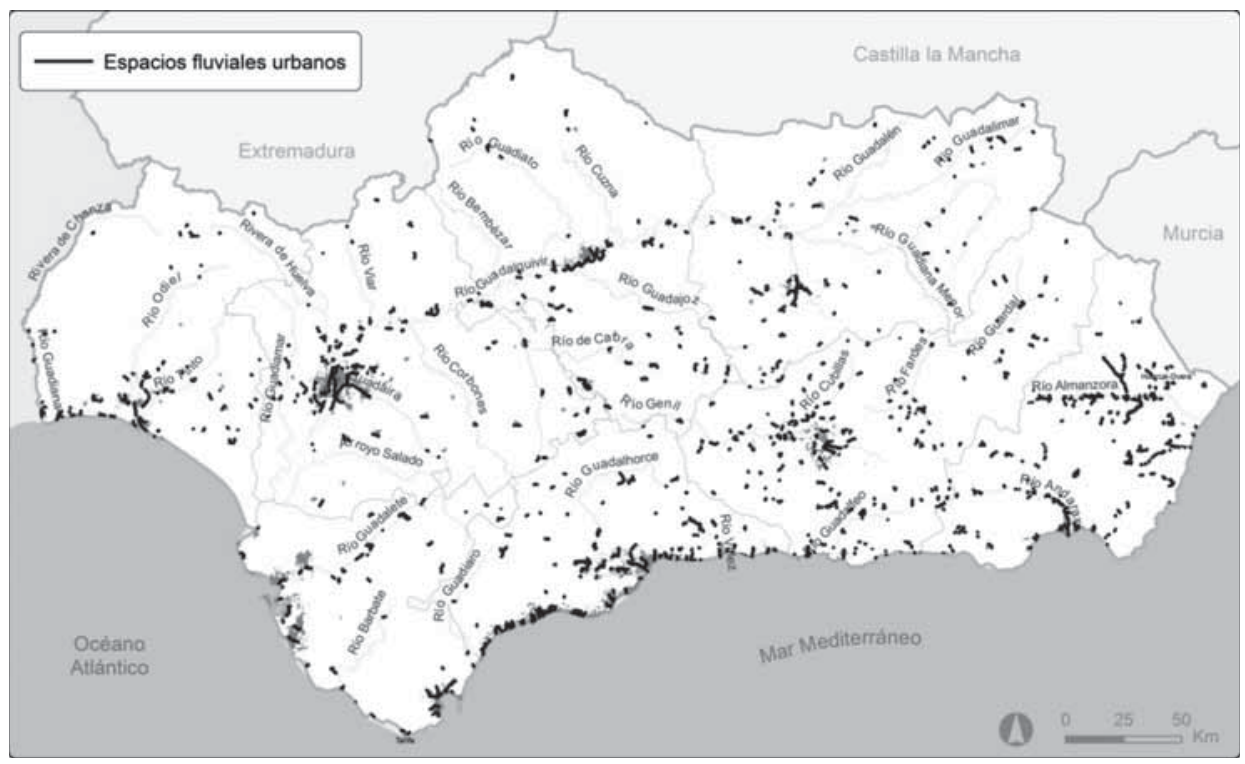

Fuente: Datos Espaciales de Andalucía para escalas intermedias (DEA100).

A continuación, se ha de considerar una escala intermedia entre lo local y lo regional, la subregional. Esta escala, además de enriquecer el análisis, resulta imprescindible para entender el territorio donde se inserta el espacio fluvial. En esta escala todavía se pueden observar los condicionantes naturales e históricos que en la escala local han sido borrados o profundamente transformados por el proceso urbanizador. Por otra parte, debido al reparto competencial actualmente vigente en la administración española, tienen gran importancia los espacios de esta escala sobre los que recae, de manera especialmente significativa para la ordenación del territorio, la actividad gestora y planificadora (Vega, 1998). Para ello, se han reconocido 
agrupaciones de espacios fluviales urbanos con características comunes: cercanía, predominancia de una categoría concreta, sistema de asentamientos, etcétera, identificando 13 ámbitos subregionales.

FIGURA 2

\section{DELIMITACIÓN DE LOS ÁMBITOS SUBREGIONALES}

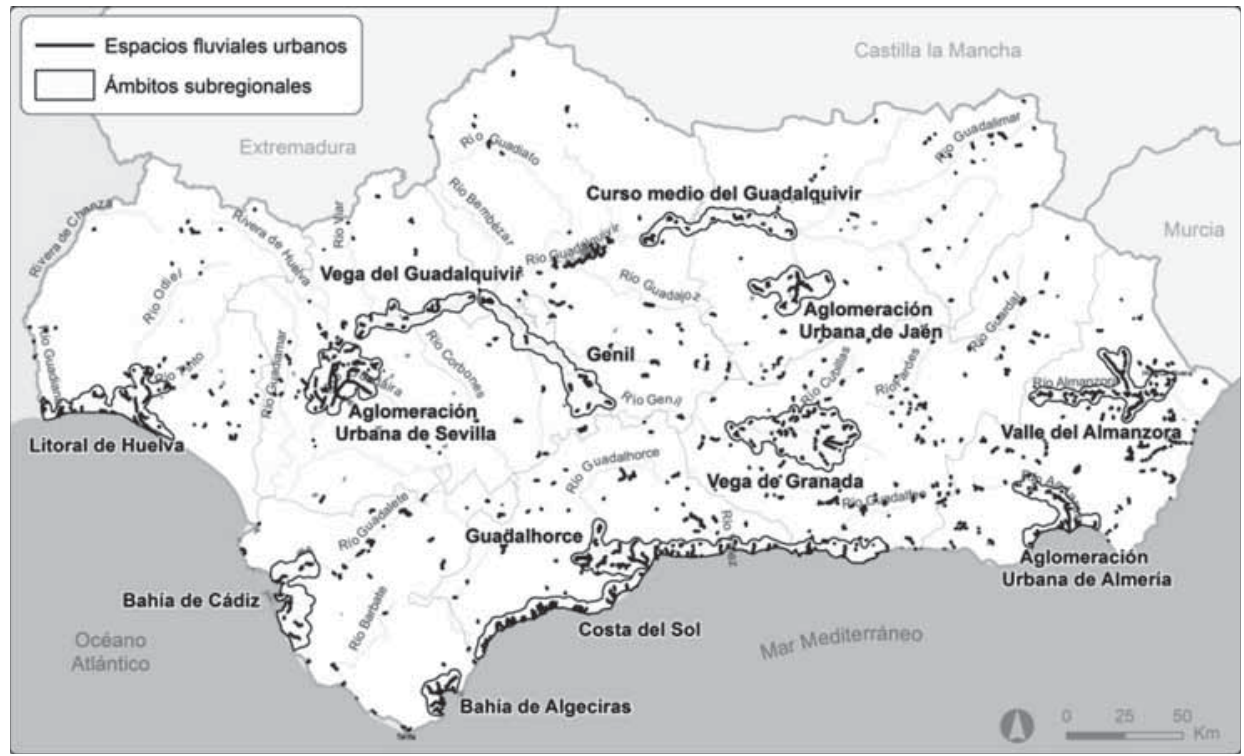

Fuente: Datos Espaciales de Andalucía para escalas intermedias (DEA100).

Las aglomeraciones urbanas de Sevilla y Almería, la Bahía de Cádiz, la Bahía de Algeciras, la Vega de Granada y el Guadalhorce son representantes de ámbitos donde el intenso proceso urbanizador ha provocado una merma de la relación río-ciudad. Estos ámbitos están afectados en mayor o menor medida por procesos de naturaleza metropolitana, lo que ha provocado la práctica desaparición, por entubamiento o desvío, de los cauces menores, prestando sólo atención a los ríos principales y sobre todo en las zonas céntricas de las ciudades.

Se distinguen diferentes situaciones de partida según la dimensión, estructura y madurez de cada ámbito urbano. Los ámbitos subregionales Aglomeración Urbana de Sevilla, Vega de Granada y Guadalhorce, responden 
a un modelo clásico de ciudad central dominante que empieza a perder peso poblacional y económico a favor de los municipios y núcleos de su primera o segunda corona metropolitana. Esto ha provocado la pérdida o alteración de los espacios fluviales de carácter periurbano. Por el contrario, en las bahías de Cádiz y Algeciras, y en la Aglomeración Urbana de Almería, aún se observan cauces y entornos fluviales periurbanos con un grado de conservación aceptable, resultado de procesos urbanísticos contemporáneos todavía no consolidados.

Los ámbitos subregionales de los valles y vegas interiores (Vega del Guadalquivir, Genil, Curso medio del Guadalquivir, Aglomeración Urbana de Jaén y Valle del Almanzora) están caracterizados por agrupaciones de ciudades medias o pequeñas en torno a un cauce principal. Los usos agrarios y los sistemas productivo locales propician una mayor relación entre la ciudad, el territorio y el espacio fluvial. El proceso urbanizador en estos ámbitos es más pausado lo que ha permitido la conservación de los principales ejes fluviales.

En el caso de los ámbitos primordialmente costeros hay que indicar que en el caso de la Costa del Sol las particularidades del proceso de urbanización (procesos expansivos que tienden a generar un continuo urbano), sumado al carácter torrencial de los ríos, han provocado la alteración de la práctica totalidad de los cauces. Por el contrario, en el Litoral de Huelva, base económica mixta de agriculturas intensivas y turismo ha permitido la conservación de muchos de los espacios fluviales.

Finalmente, la escala local es el ámbito más acotado de análisis, donde la complejidad de la realidad territorial alcanza aquí su máxima expresión; la relación entre lo natural y lo construido se ve condicionada por peculiaridades que singularizan cada lugar (Zevi, 1995). El principal condicionante de esta escala es el proceso urbanizador, que prevalece sobre los condicionantes naturales e históricos.

La segunda concepción que se ha de tener en cuenta es la escala temporal, siendo la evolución del espacio fluvial a lo largo del tiempo una dimensión fundamental para su estudio, profundizando en el crecimiento y la evolución de las ciudad respecto al espacio fluvial, utilizando para ello tanto fuentes escritas, principalmente planes urbanísticos, como documentación cartográfica y fotográfica. Con frecuencia se trata de explicar el crecimiento de la ciudad, como un desarrollo a saltos y de partes inconexas. Sin embargo, el proceso urbanizador y el tratamiento que se le ha dado a los espacios fluviales es consecuencia de la historia urbana y de los acontecimientos políticos, económicos y culturales que se suceden en cada lugar. 


\subsection{Caracterización del espacio fluvial urbano.}

Una vez identificadas las distintas escalas de análisis se ha de realizar un estudio de las relaciones entre los dos elementos considerados, espacio fluvial y espacio urbano, basado en tres variables fundamentales: la dinámica del río, su grado de intervención y la posición de la trama urbana respecto al espacio fluvial.

La interrelación entre estas variables da lugar a los distintos tipos de espacio fluvial urbano que sirven para explicar la forma resultante de la interacciones entre río y ciudad. A través de la consideración de las variables mencionadas se trata de establecer hasta qué punto existen procesos comunes y especificidades en cada uno de los entornos estudiados.

\subsubsection{Dinámica del espacio fluvial.}

Uno de los motivos por los cuales las relaciones río-ciudad resultan tan diversas es la dinámica fluvial, condicionada por la singularidad y variedad de las escenarios naturales (clima, litología, geología, altitud, latitud, relieve, temperatura, etcétera) y antrópicas (regulación de caudales, actuaciones sobre el cauce, desvíos, bombeos, etcétera). El período durante el cual el río lleva agua en su cauce resulta de gran importancia tanto para las comunidades vegetales y animales, como para el desarrollo de los distintos usos en las márgenes. Por tanto, el crecimiento urbano se verá influido por la presencia de una lámina de agua más o menos constante a lo largo del año.

En el caso de Europa, los ríos son moderadamente largos, sin grandes avenidas y, en general, regulares. La excepción a estas características son los ríos mediterráneos que, siendo más cortos, tienen profundos estiajes y grandes avenidas, lo cual explicaría las dificultades de integración en el caso de las ciudades españolas respecto a las ciudades septentrionales o centroeuropeas (Monclús, 2002).

Sin embargo, no es posible establecer tipologías de interacciones entre ciudades y ríos solo en función de las características naturales de los mismos pues, tal y como señalaba ya Buffon en su Historia Natural escrita en el siglo XVIII, «los ríos que conocemos no son solo obra de la naturaleza sino del esfuerzo de los hombres y de las sociedades avanzadas»². El proceso de

2 Referencia al artículo sobre ríos de la Historia Natural de Buffon, citado por C.J. Glacken en Huellas en la playa de Rodas, Ed. del Serbal, Barcelona, 1996, pág. 623. 
regularización progresiva de los ríos, sobre todo a su paso por los núcleos de población, constituye un aspecto fundamental cuando se trata de explicar su configuración actual. Por ello, es necesario incluir dentro del estudio histórico de la evolución del cauce los componentes que modifican la dinámica natural. Por ejemplo, el actual carácter temporal del río Guadalmedina a su paso por Málaga no se puede entender sin remontarse a las deforestaciones del siglo XVI para la plantación de viñedos y aprovechamiento de madera o a la construcción en el decenio de los ochenta de la presa de El Limonero.

Una vez hecha la puntualización anterior, se puede diferenciar entre espacios fluviales urbanos con dinámica permanente o con dinámica temporal. Los primeros, están caracterizados por mantener una lámina de agua constante durante todo el año, aunque con la posibilidad de tener pequeñas variaciones de caudal a lo largo del mismo. En estos casos, la presencia continua de agua, bien sea por causas naturales o por la regularización del río, define unos límites más o menos estables del cauce, lo que ha permitido un mayor acercamiento de la ciudad al espacio fluvial. En general estos cauces no son vadeables, por lo que son necesarios puentes y otras estructuras para cruzar de una orilla a otra.

Por el contrario, los espacios fluviales urbanos con dinámica temporal se caracterizan por una lámina de agua variable, permaneciendo secos parte del año o durante sequías prolongadas, aunque pueden existir pozas con agua o flujos intermitentes. Estos ríos se incluirian en las categorías de ríos efímeros, ríos intermitentes o fuertemente estacionales o ríos temporales o estacionales de la Orden ARM/2656/2008, de 10 de septiembre, por la que se aprueba la instrucción de planificación hidrológica, todos con un periodo de flujo de agua menor de 300 días al año. Las ramblas, características de las cuencas mediterráneas, son ríos efímeros y sus cauces suelen ser muy anchos y en ocasiones difíciles de delimitar. En estos casos, el régimen irregular provoca, en la mayoría de las ocasiones, un mayor alejamiento de la ciudad respecto al río.

El tipo de dinámica fluvial también tiene influencia en el grado de intervención que ha sufrido el cauce. La dinámica temporal de muchos ríos de la región mediterránea ha condicionado las grandes intervenciones realizadas que en algunos casos. A pesar del esfuerzo y la técnica humana, dificilmente han logrado ordenar los cauces fluviales.

Pese a que en Andalucía prevalecen los ríos o tramos de ríos temporales, según el Plan director de riberas de Andalucía (Consejería de Medio Ambiente, 2003) los tramos temporales o esporádicos representan el $57 \%$ y los tramos permanentes el $43 \%$, en lo referido a espacios fluviales urbanos esa predominancia no es tan clara. Los espacios fluviales urbanos con dinámica temporal representan el 50,14\%, frente al 49,86\% de los permanentes. Esta 
menor diferencia es debida a la ventaja que la dinámica permanente ofrece al crecimiento urbano, por lo que muchos núcleos andaluces se asientan en las proximidades de este tipo de tramos.

Desde el punto de vista de la dinámica fluvial, la red hidrográfica andaluza puede dividirse en dos grandes zonas. Por un lado se encuentra la zona con dinámica fluvial temporal, constituida por un conjunto de ríos y arroyos que nacen en sierras del Sistema Bético y desembocan en el mar Mediterráneo, así como por los afluentes que bajan de Sierra Morena. En este contexto hidrográfico se encuentran cauces que presentan frecuentemente una morfología de rambla en los tramos medios y bajos, presentando en general un régimen de caudales caracterizado por su gran variabilidad (valles del Almanzora, del Andarax, del Guadalhorce, Cabo de Gata, etcétera). También destacan los innumerables arroyos de fuerte pendiente, corto recorrido y aportes esporádicos perpendiculares al litoral (Costa del Sol y Costa de Granada), al sur, y los pequeños arroyos de aguas pluviales de la Sierra de Aracena, Sierra Morena, Los Pedroches y Despeñaperros, al norte.

Por otro lado, la zona con dinámica fluvial permanente engloba el litoral atlántico y los grandes valles interiores. En las cuencas del litoral atlántico la topografía permite que se desarrollen amplias zonas de marismas, asociadas a los estuarios de los ríos Barbate, Guadalete, Guadalquivir, Tinto y Odiel. La alta regulación de estos ríos, junto con los aportes de los flujos de marea permite la presencia de agua permanentemente. También se encuentran dentro de esta zona los grandes ríos de los valles interiores (Guadalquivir, Genil, Guadiana Menor, Guadalimar, etcétera), caracterizados por la alta regulación de sus cuencas.

\subsubsection{Intervención sobre el espacio fluvial.}

En origen, la relación río-ciudad estaba marcada por el carácter irregular natural del espacio fluvial. Con el paso del tiempo y el avance de la técnica, la actitud contemplativa-pasiva cambió a otra de dominio de la ciudad sobre el espacio fluvial, donde predominan los componentes artificiales sobre los naturales. Por lo tanto, se parte de la base de que, en el ámbito urbano en el que se enmarca el presente trabajo, es común algún tipo de intervención sobre el cauce a lo largo de la história. El objetivo de esta variable es diferenciar el grado de intervención, es decir, si la alteración resultante es alta o baja.

Se ha considerado que un espacio fluvial urbano está alterado cuando la intervención sobre el mismo es alta, es decir, se han realizado grandes obras 
de defensa, desplazamientos del cauce, entubamientos, etcétera. Además, ha desaparecido la práctica totalidad de la vegetación de ribera. Por el contrario, cuando no se ha intervenido sobre el espacio fluvial o solo se han realizado pequeñas obras de defensa o estabilización del cauce que permiten parte de la dinámica natural del río, se considera que el espacio fluvial urbano está poco o nada alterado.

El proceso de ocupación y uso de los espacios fluviales urbanos, y por consiguiente la posición de la ciudad respecto al río, se vincula a las dos variables anteriores. Por un lado, los cauces regulares con dinámica permanente o intervenidos permiten una mayor cercanía y el crecimiento en las márgenes. Por otro lado, los cauces irregulares con dinámica temporal o poco intervenidos limitan el crecimiento de la ciudad.

A partir de la segunda mitad del siglo XX se ha desarrollado en Andalucía un proceso caracterizado por una profunda alteración de las condiciones naturales y una tendencia al incremento continuado de la superficie urbana, sobre todo en el litoral. Al igual que en el resto de regiones, en Andalucía la distribución espacial de las intervenciones sobre el espacio fluvial urbano está condicionada por dos factores principales. Por un lado, a mayor variabilidad de caudales, mayor es la posibilidad de que ocurran avenidas e inundaciones y, por lo tanto, es necesario un mayor control del cauce. Por otro lado, cuanto mayor es la población y el crecimiento urbano, mayor es la necesidad de defenderse.

En general, los espacios fluviales andaluces están poco intervenidos. En su mayor parte se han realizado intervenciones que pueden considerarse de pequeño tamaño como motas o muros de contención. Solo en las principales ciudades se han realizado grandes obras de defensa, desplazamientos del cauce o canalizaciones. Se ha identificado, mediante fotointerpretación, un total de $294,60 \mathrm{~km}$ de espacios fluviales urbanos considerados como alterados que representan el 13,23\% del total. Éstos están distribuidos principalmente en torno a las grandes aglomeraciones urbanas (Córdoba, Granada, Málaga y Sevilla) y en el Poniente Almeriense. El resto, 1.932,29 km, un 86,77\%, se han considerado como poco o nada alterados.

\subsubsection{Posición: lejanía, desarrollo asimétrico y centralidad.}

No se puede disociar la posición actual que ocupa la ciudad respecto al espacio fluvial del proceso histórico de crecimiento urbano. En su origen, las ciudades que se ubicaban próximas a un curso de agua lo hacían a cierta distancia del mismo. Normalmente, aprovechaban las terrazas formadas por 
los depósitos sedimentarios, debido a la protección que ofrecían frente a las crecidas del río. Otro elemento natural que ofrecía ventajas a la hora de instalar ciertos asentamientos históricos, son los meandros, sobre todo en los tramos medios de baja pendiente, donde el efecto coriolis y la litología propician estas formaciones. El caso de la ubicación de Montoro es buen ejemplo de ello.

Con el avance de la técnica y el mejor control de cauce, el espacio urbano fue extendiéndose hacia las proximidades del río estableciendose una relación entre el tamaño de la ciudad y la escala de un cauce fluvial. Así en las ciudades de pequeño tamaño el río adquiere mayor amplitud, mientras en las ciudades con mayor crecimiento el río discurre en un curso de menor envergadura. El establecimiento de esta relación entre el espacio urbano y el fluvial da cuenta del carácter interdependiente de ambos, y de cómo el crecimiento y desarrollo de la ciudad ejerce un dominio sobre su medio natural. La aproximación de la ciudad al río se producía en un primer momento de forma asimétrica, dadas las dificultades para atravesar el río. Posteriormente, la construcción de puentes hizo posible el salto a la otra margen.

Para el presente trabajo, se han considerado tres tipos de posición de la trama urbana respecto al espacio fluvial. En la posición central el espacio fluvial ocupa una posición centrada dentro del entorno urbano; existe una clara relación y continuidad entre las dos márgenes. En la posición asimétrica o tangencial, el emplazamiento y crecimiento urbano histórico se localizan en una sola margen, aunque pueden existir crecimientos en la opuesta, estos están desligados de la ciudad central y los usos son distintos a los residenciales; no existe continuidad entre ambas márgenes. Por último, cuando el espacio fluvial ocupa una posición alejada del entorno urbano se considera que tiene una posición lejana, aunque pueden existir edificaciones aisladas entre el espacio urbano y el cauce.

En la actualidad, como norma general, los núcleos de población andaluces se localizan a cierta distancia de sus cauces. Se han identificado mediante fotointerpretación 1.685,99 km de espacios fluviales alejados, lo que representa el 75,71\%, distribuidos principalmente por las provincias de Almería, Granada y Málaga. Los espacios fluviales con posición asimétrica o tangencial suman $400,11 \mathrm{~km}$, el $17,97 \%$ del conjunto de tramos identificados. Las provincias de Sevilla, Córdoba y Huelva son las que cuentan con mayor número de kilómetros de espacios asimétricos. Por último, los espacios fluviales centrales solo suman 140,80 km, el 6,32\% de todos los tramos identificados.

A través de la consideración de las variables mencionadas se trata de establecer hasta qué punto existen procesos comunes y especificidades en cada uno de los casos estudiados. 


\subsection{Incorporación de la dimensión patrimonial y la identidad}

En la vertiente patrimonial, los espacios fluviales urbanos se caracterizan también por el importante patrimonio cultural que debe ser valorado y conservado. En estos entornos destacan numerosos recursos patrimoniales, que son testigos de un importante intercambio de valores humanos y del desarrollo armonioso de interacciones entre los hombres y su medio ambiente a lo largo de la historia (ICOMOS, 2009).

Los espacios fluvio-urbanos juegan un importante papel en la construcción de las identidades de los individuos, grupos y comunidades urbanas. Focalizan y articulan el tejido urbano, aportando significados e imágenes de la ciudad, haciéndola reconocible, sin posibilidad de confundirla con otra. Se trata de algo más que el escenario físico donde se desarrolla parte de la vida de la ciudad, siendo un producto fruto de la interacción simbólica que se da entre las personas que comparten un determinado entorno urbano.

La mayoría de las ciudades han nacido y crecido entorno a un cauce, por lo que son numerosos los elementos patrimoniales históricos (patrimonio material) y también son frecuentes las tradiciones de prácticas sociales (romerías o fiestas) ligadas al río (patrimonio inmaterial; González de Tánago y García Jalón, 2007).

Los citados valores patrimoniales refuerzan el aprecio y la atribución de significados a los cursos fluviales, constituyéndose en muchos casos en referentes para promover actuaciones de recalificación y puesta en uso público de determinados itinerarios o espacios ribereños (Zoido, Rodríguez, Ramírez, 2010).

Por otro lado, los espacios fluviales urbanos son una clara expresión territorial de la forma en que se relaciona una sociedad concreta con su medio. En este sentido, el patrimonio es un elemento esencial desde el que valorar la identidad del espacio fluvial urbano, lo que justifica un esfuerzo de renovación y de puesta en valor. La mayoría de las actuaciones en cauces urbanos han generado transformaciones rápidas e intensas que provocan la pérdida de esta identidad, lo que significa una merma para la ciudad.

Así pues, la atención a los espacios fluviales urbanos no tiene sentido si no se desarrolla una aproximación desde la identidad que tenga como base, tanto la observación de la interacción de los elementos naturales y construidos a lo largo del tiempo, como el estudio de las representaciones de los individuos y grupos sociales que viven y disfrutan de esos entornos. Además, el estudio histórico-patrimonial debe servir para prever mejor el futuro de estos lugares y plantear posibles políticas o acciones. 
A la hora de incorporar la dimensión patrimonial al estudio de los espacios fluviales urbanos es necesario realizar en primer lugar un análisis de la evolución histórica de las relaciones río-ciudad, utilizando para ello tanto fuentes escritas, principalmente planes urbanísticos, como documentación cartográfica y fotográfica. Con frecuencia se trata de explicar el crecimiento de la ciudad, como un desarrollo a saltos y de partes inconexas. Sin embargo, el proceso urbanizador y el tratamiento que se le ha dado a los espacios fluviales es consecuencia de la historia urbana y de los acontecimientos políticos, económicos y culturales que se suceden en cada lugar.

Una vez analizada la evolución histórica de la ciudad y el espacio fluvial, se estudian los distintos elementos que configuran el soporte físico de la trama urbana. Estos elementos se desglosan en espacios públicos (viario y espacios libres) y espacios edificados, los cuales a su vez pueden tener un uso público o privado.

Por otro lado, se analizan los edificios y elementos singulares de interés, bien histórico o bien arquitectónico merecedores de algún tipo de protección legal. Los espacios fluviales urbanos que poseen gran número de elementos patrimoniales se hacen reconocibles, incluso por personas que no son de la ciudad. Así, se estudian las edificaciones declaradas como bien de interés cultural o incluidas en catálogos o listados que forman parte del planeamiento urbanístico. Igualmente se ha de tener en cuenta las agrupaciones de estos elementos, como el estudio de los conjuntos históricos.

Posteriormente, se ha de realizar un examen de las percepciones sociales orientado fundamentalmente a la identificación de las diversas miradas que la sociedad, representada a través de sus distintos agentes, expresa con relación al río y al espacio urbano a lo largo del tiempo. Este estudio se ha realizado a través del análisis de representaciones artísticas y culturales, que con frecuencia recogen la información acumulada en el imaginario colectivo. En este sentido, se han analizado los valores y significados atribuidos al espacio fluvial urbano a través de fuentes literarias (poesía, relatos de viajes, etcétera), expresiones artísticas, logotipos, guías y folletos turísticos, fotografías históricas y postales, etcétera. Estas representaciones permiten confirmar las relaciones identitarias que se establecen entre la sociedad y el espacio fluvial urbano, así como representar una valiosa fuente para conocer mejor las transformaciones urbanas. Este análisis de las representaciones literarias y artísticas permite comparar el tratamiento y las singularidades de cada espacio fluvial urbano a lo largo del tiempo. 


\section{Conclusiones}

Con el presente trabajo se pone de manifiesto la necesidad de trabajar en distintas escalas, espaciales y temporales, y el reconocimiento de las relaciones entre los ríos y el patrimonio a la hora del estudio de los espacios fluviales urbanos para poder entender su identidad y carácter. Por lo tanto, el presente trabajo incluye dos nuevos conceptos a la hora del estudio de los espacios fluviales urbanos que hasta ahora no había sido estudiados de forma conjunta en Andalucía: el análisis en distintas escalas y el estudio de los aspectos materiales e inmateriales.

La incorporación de la escala subregional: se trata de la escala de referencia por excelencia, no sólo porque representa una buena oportunidad para aproximarse a cada una de las tipologías analizadas en el presente trabajo, sino porque supone también la consideración del espacio fluvial urbano, tanto en las políticas de ordenación del territorio, como en las de medio ambiente, patrimonio, etcétera. Por lo tanto, la escala subregional resulta imprescindible para armonizar entre sí distintos tratamientos que realizan los planes de alcance municipal sobre los espacios fluviales.

Incorporar la escala intermedia como una variable más en el análisis y valoración de los espacios fluviales urbanos supone, por tanto, favorecer la conservación y mejora de estos entornos, y planificar de manera adecuada la transformación de los usos del suelo.

Tal y como se ha comentado anteriormente, se considera que el análisis en distintas escalas, temporales y espaciales, es determinante para poder entender las transformaciones de los espacios fluviales urbanos, ya que son resultado de múltiples procesos que actúan sobre distintas escalas.

Asimismo, para la caracterización de los espacios fluvio-urbanos es necesario realizar una descripción y catalogación de los elementos, tanto materiales (naturales, urbanísticos y culturales) como inmateriales (percepciones sociales, representaciones, etcétera), que les otorgan identidad. En este sentido, en vez de separar el espíritu y el lugar, lo inmaterial y lo material y colocarlos como elementos opuestos, es necesario examinar las relaciones en las que los dos interactúan y se construyen recíprocamente.

Los espacios fluvio-urbanos juegan un importante papel en la construcción de las identidades de los individuos, grupos y comunidades urbanas. Focalizan y articulan el tejido urbano, aportando significados e imágenes de la ciudad, haciéndola reconocible, sin posibilidad de confundirla con otra. Se trata de algo más que el escenario físico donde se desarrolla parte de la vida de la ciudad, siendo un producto fruto de la interacción simbólica que se da entre 
las personas que comparten un determinado entorno urbano. Es por ello que muchas ciudades andaluzas tengan un claro rasgo de su personalidad en los ríos que las atraviesan, siendo estos protagonistas no solo de su imagen o su historia, sino que forman parte de su propia esencia como ciudad.

Por otro lado, los espacios fluviales urbanos son una clara expresión territorial de la forma en que se relaciona una sociedad concreta con su medio. En este sentido, el patrimonio es un elemento esencial desde el que valorar la identidad del espacio fluvial urbano, lo que justifica un esfuerzo de renovación y de puesta en valor.

Es numeroso el patrimonio material e inmaterial ligado a los espacios fluviales urbanos en las ciudades andaluzas. Igualmente importante es el patrimonio local relacionado con el río no inventariado oficialmente. Es sustancial dar a conocer estos elementos, a menudo olvidados o deteriorados. La vinculación de ciudades como Sevilla y Córdoba con el Guadalquivir o la que establece Granada con el Genil y sus afluentes de cabecera, así como las que se aprecian en Alcalá de Guadaíra, Écija, Coria del Río, etcétera, constituyen ejemplos significativos de los valores y potencialidades patrimoniales que se derivan de las relaciones entre los núcleos urbanos andaluces y los espacios fluviales.

La mayoría de las actuaciones en cauces urbanos han generado transformaciones rápidas e intensas que provocan la pérdida de esta identidad, lo que significa una merma para la ciudad. Con este trabajo se puede confirmar que muchos de los espacios fluvio-urbanos andaluces son entornos de carácter patrimonial. Por lo tanto, es importante que las acciones que se tengan en cuenta instituciones relacionadas con la conservación del patrimonio. La recuperación de las riberas fluviales urbanas es una oportunidad para devolver la naturalidad del paisaje y aprovechar las energías del sistema natural, así como mantener y potenciar el patrimonio cultural.

Así pues, la atención a los espacios fluviales urbanos no tiene sentido si no se desarrolla una aproximación desde la identidad que tenga como base, tanto la observación de la interacción de los elementos naturales y construidos a lo largo del tiempo, como el estudio de las representaciones de los individuos y grupos sociales que viven y disfrutan de esos entornos. Además, el estudio histórico-patrimonial debe servir para prever mejor el futuro de estos lugares y plantear posibles políticas o acciones.

Esta metodología, por su amplitud y carácter, encara de forma unitaria y multidisciplinar los espacios fluvio-urbanos, con el objetivo de analizar estos espacios como garantía de su conservación y puesta en valor dentro de su ámbito urbano. 


\section{BiBLiOgRAFÍA}

Amoros, C., Petts, G. E. (1993): Hydrosystemse fluviaux. Paris-Milan-BarcelonneBonne: Masson.

Bravard, J.-P., Laurent, M. A., Davalon, J. Y Bethemon, J. (1995): Las paysages d'eau aux portes de la ville. Programme Rhône - Alpes. Recherches en Science Humaines. Centre Jacques Cartier. ISBN 2 909604-18-7

Calvo García-Tornel, F. (1997): "Las transformaciones de los espacios urbanos fluviales en zonas áridas" en Documents d’Anàlisi Geografica, n 31, págs. 103-116.

Carmona González, P. (1997): "La dinámica fluvial del Turia en la construcción de la ciudad de Valencia” en Documents d'Anàlisi Geografica, no 31, págs. 85-102.

Consejería de Medio Ambiente (2003): Plan Director de Riberas de Andalucía (Documento borrador).

Díaz del Olmo, F., Almoguera Sallent, P. (coords.) (2013): Sevilla, la ciudad y la riada del Tamarguillo (1961). Serie Historia y Geografía, no 252. Secretariado de Publicaciones, Universidad de Sevilla.

Glacken, C.J. (1996): Huellas en la playa de Rodas. Barcelona: Editorial del Serbal, pág. 623.

García García, A. (2002): "Potenciales de la rehabilitación de cursos fluviales en el marco de los nuevos modelos urbanos. El ejemplo del arroyo Tagarete en Sevilla". Comunicación III Congreso Ibérico sobre Gestión y Planificación de Aguas, La Directiva Marco del Agua: Realidades y Futuros. Sevilla.

González de Tánago, M., García Jalón, D. (2007): Restauración de ríos. Guía metodológica para la elaboración de proyectos. Ministerio de Medio Ambiente, pág. 202.

González Fustegueras, M. A., Lastra Valdor, I. (2007): La urbanización y su efecto en los ríos. Documento Mesas de Trabajo de la Estrategia Nacional de Restauración de ríos. Ministerio de Medio Ambiente, Medio Rural y Marino.

International Council on Monuments and Sites (ICOMOS) (2009): World Heritage Cultural landscapes. UNESCO-ICOMOS. Documentation Centre, pág. 213.

Leopold, W., Wolman, M. G. (1957): River channel pattern-braided, meandring and straight. US Geol. Surv. Prof. Paper, págs. 282.

Monclús Fraga, F. J., (2002): "Ríos, ciudades, parques fluviales, corredores verdes" en Ríos y Ciudades. Aportaciones para la recuperación de los ríos y riberas de Zaragoza, coord. de la Cal, P. y Pellicer, F., Institución Fernando el Católico, págs. 11-31.

Moral Ituarte, L. (1997): "El agua en la organización del espacio urbano: El caso de Sevilla y el Guadalquivir" en Documents d'Anàlisi Geografica, nº 31, págs. 117-127.

Ollero Ojeda, A., Ureña Francés, J. M., (2000): "Criterios y propuestas para la ordenación de áreas fluviales" en Ciudad y territorio: Estudios territoriales, no 126 , págs. 689-710.

Ollero, A. (2007): Territorio Fluvial. Diagnóstico y propuesta para la gestión ambiental y de riesgos en el Ebro y los cursos bajos de sus afluentes. Bakeaz/Fundación Nueva Cultura del Agua, Bilbao. 
Ollero, A., Ibisate, A., González De Matauco, V., Naverac, A., Díaz, E., Granado, D., García, J. H. (2011): "Innovación y libertad fluvial”. Comunicación VII Congreso Ibérico sobre Gestión y Planificación del Agua.

Pellicer, F, (2002): "Ordenación paisajística de espacios fluviales en las ciudades mediterráneas", en Zoido, F. y Venegas, C., Paisaje y ordenación del territorio. Consejería de Obras Públicas y Transportes. Sevilla.

Riley, A. L. (1998): Restoring streams in cities: a guide for planners, policy makers, and citizens. Island Press, Washington D.C.

Sala, M., Inbar, M. (1992): "Some hydrologic effects of urbanization in Catalan rivers" en Catena, $\mathrm{n}^{\circ} 19$, págs. 363-378.

Terrin J.-J. (2014): Villes inondables, Prévention, adaptation, résilience. Marseille, Parenthèses.

Ureña, J. M. (2000): "La urbanización de los ríos o la conservación de la naturaleza en las ciudades: Tipología de actuaciones" en Los Ríos y las Ciudades. Actas, Conferencia Regional del Agua, págs. 131-151.

Vannote, R.L., Minshall, G.W., Cmins, K.W. et al. (1980): "The river continuum concept" en Canad. J. of Fisheries and Aquatic Sc., n 37, págs. 130-137.

Vega González, G. (1998): "La ordenación territorial de escala comarcal" en Boletín de la AGE, $\mathrm{n}^{\circ}$ 26, págs. 161-176.

Wade, M. (1997): "Actuacions de rehabilitació fluvial en medis urbans. Els casos de Manchester i Londres (Regne Unit) i Phoenix i Tempe (Estats Units)" en Documents d’Anàlisi Geografica, nº 31, págs. 35-56.

Zevi, B. (1995): Paesaggi e cittá. Newton Compton, Roma.

Zoido Naranjo, F., Rodríguez Rodríguez, J., Ramírez Ramírez, A., (2010): Los paisajes fluviales en la Cuenca del Guadalquivir: Instrumentos Básicos para su consideración en la Planificación y Gestión Hidrológica. Ministerio de Medio Ambiente, Medio Rural y Marino, Confederación Hidrográfica del Guadalquivir.

Fecha de recepción: 5 de octubre de 2016.

Fecha de aceptación: 15 de junio de 2017. 